J. Gynäkol. Endokrinol. AT 2019 · 29:6-12 https://doi.org/10.1007/s41974-019-0083-y Online publiziert: 5. April 2019

(c) Der/die Autor(en) 2019

\section{Kazem Nouri}

Universitätsklinik für Frauenheilkunde, Medizinische Universität Wien, Wien, Österreich

\title{
Medikamentöse Therapie des Uterus myomatosus mit dem Präparat Ulipristalacetat - ein Update
}

Myoma uteri sind die am häufigsten auftretenden gutartigen Tumoren des Uterus. Die Daten sprechen davon, dass zwischen 20 und $50 \%$ der Frauen im reproduktionsfähigen Alter von Myomen betroffen sind, wobei nahezu bei jeder zweiten Diagnose klinische Symptome mit einhergehen. Bei Unterscheidung der Ethnizität hat die Mehrheit der Patientinnen nur ein Myom, dessen Durchmesser in fast der Hälfte der Fälle 1-2 cm beträgt. Weiter ist die Mehrheit der Myome intramural und subserös lokalisiert, wobei Anzahl und Größe bis zum Einsetzen der Menopause zunehmen [1].

\section{Pathogenese}

Gegenwärtig ist der Entstehungsmechanismus der Myome nicht vollständig geklärt, man geht jedoch davon aus, dass aus einer einzelnen physiologischen Muskelzelle durch noch nicht näher bekannte Faktoren eine pathologische Muskelzelle entsteht. Durch Proliferation dieser abnormen Muskelzelle entstehen unter dem Einfluss von Hormonen mehrere entartete Muskelzellen. Dieser Vorgang wird als eine klonale Expansion [2] bezeichnet und man weiß heute, dass Östrogene und Progestagene eine wesentliche Rolle bei der Entstehung der Myome spielen. Allerdings zeigt die Tatsache, dass es bei einer Patientin mit mehreren Myomen durchaus möglich ist, dass manche Myo-

Aus Gründen der besseren Lesbarkeit wird in dieser Fachpublikation in der Regel das generische Maskulinum als geschlechtsneutrale Form verwendet. me wachsen und gleichzeitig andere an Größe abnehmen [3, 4], eindeutig, dass andere zusätzliche Faktoren das Wachstum der Myome beeinflussen.

Daher ist der Schluss zulässig, dass dieses Phänomen die Verhaltensabhängigkeit von Myomen von lokalen Faktoren („growth factors“ und „apoptosisrelated factors"; - Abb. 1) linear bekräftigt. Durch präklinische Studien konnte gezeigt werden, dass die Konzentration von Progesteronrezeptor A und Progesteronrezeptor B innerhalb von Myomen deutlich höher ist als im umliegenden myometralen Gewebe. Weiters sind die mitotischen Aktivitäten sowie die Konzentration von EGF-mRNA in der zweiten Zyklushälfte, also in der Progesterondominanz, deutlich höher im Vergleich zur ersten Zyklushälfte. Bekannt ist auch die Tatsache, dass BCI-2-Proteine in Uterusleiomyomen durch Progesterone abreguliert werden [5-8].

\section{Symptome}

Die durch Myome verursachten klinischen Symptome sind von ihrer Lokalisation und ihrer Größe abhängig (• Abb. 2).

Die intramuralen Myome stellen mit $50 \%$ die häufigste Gruppe dar. Sie verursachen hauptsächlich Blutungsstörungen im Sinne von Hypermenorrhö, Dysmenorrhö und Menorrhagie. Zusammen mit den subserösen Tumoren, die 35\% der Myome ausmachen, können sie durch Druck auf die Nachbarorgane Symptome wie Verstopfung, Miktionsbeschwerden und selbst Rückenschmerzen auslösen. Die submukösen Myome haben einen nur 5\%igen Anteil und verursachen hauptsächlich Blutungsstörungen, die eventuell $\mathrm{zu}$ einer sekundären Anämie führen können. Außerdem sind sie verantwortlich für die Herabsetzung der Implantationsrate und vermindern somit die Fruchtbarkeit einer Frau [9-11].

\section{Myome und Infertilität}

Myome stellen bei einer geringen Anzahl (ca. 1-2,4\% der Patientinnen) die alleinige Ursache für Infertilität und Sterilität dar. Bei Patientinnen mit unerfülltem Kinderwunsch werden zusätzlich bei ca. 5-10\% Myome diagnostiziert [12].

Es gibt einige Hypothesen, die die negative Beeinflussung der Fertilität durch die Myome zu erklären versuchen. Die Myome können durch ihre Lage und Größe zu mechanischen Obstruktionen, einer Störung der subendometrialen Kontraktionen und einer verminderten $\mathrm{Be}$ reitschaft des Endometriums für die Aufnahme des Embryos führen [13].

Das Letztere ist vor allem bei den submukösen Myomen und intramuralen Myomen, die bis zu einer Deviation des Cavum uteri führen, der Fall [14].

\section{Therapie}

Ein wesentliches Element bei der Behandlung der Myome ist auch weiterhin der Grundsatz, dass eine Therapie bei Auftreten von Symptomen und deren raschem, großem Zuwachs rasch einsetzen muss. Bei Patientinnen mit diagnostiziertem Myom, bei denen diese Argumente nicht zutreffen, reichen Ultraschallunter- 


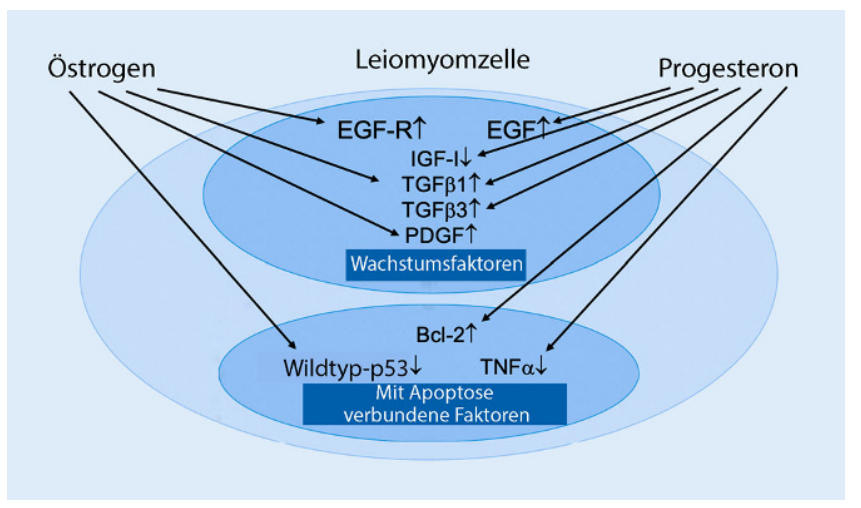

Abb. $1<$ Lokale Faktoren, die für das Abnehmen oder Zunehmen der Größe derMyome eine Rolle spielen

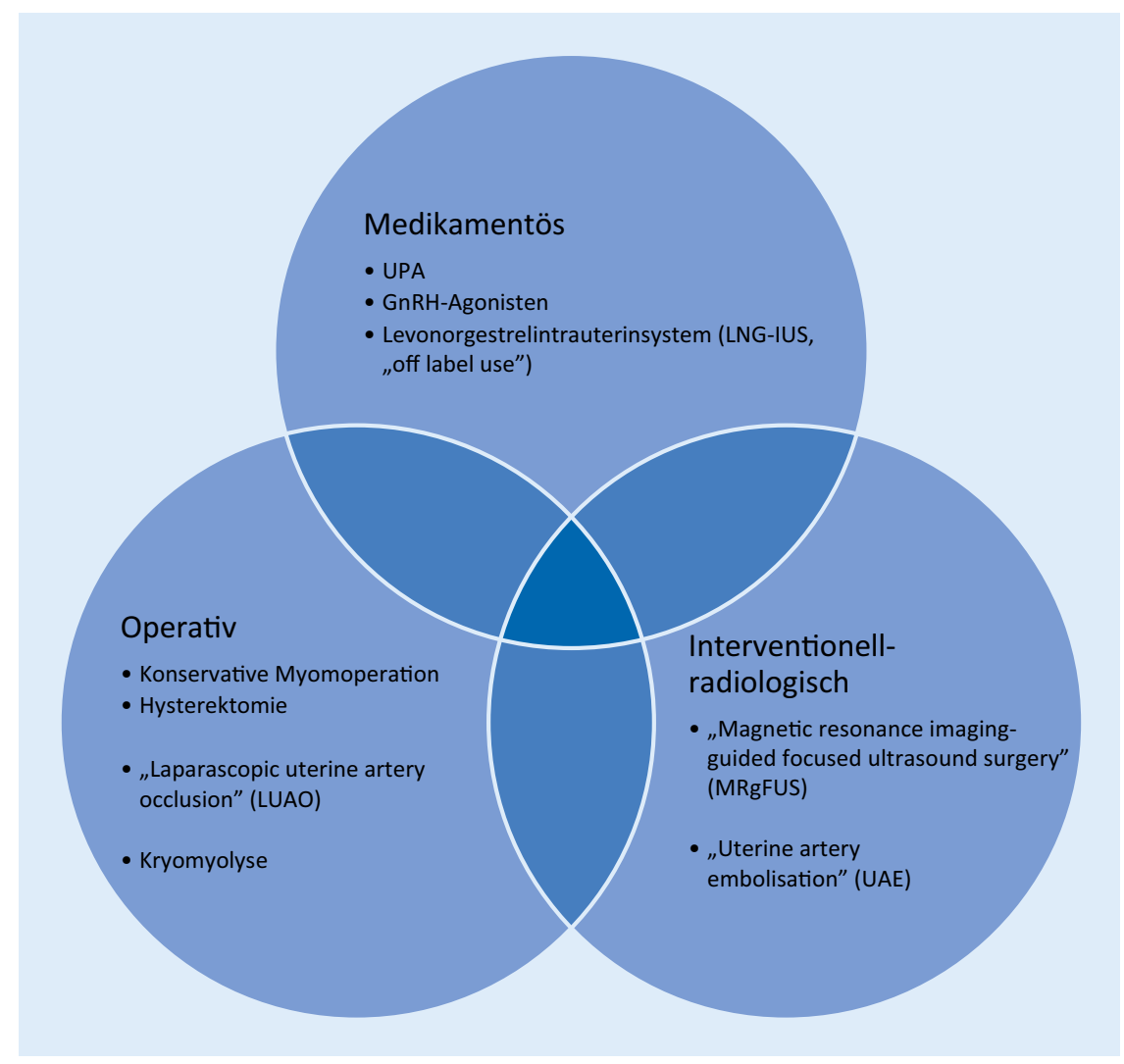

Abb. 3 \ Therapieoptionen für die Behandlung von Myomen

suchungen in einem Intervall von $6 \mathrm{Mo-}$ naten aus.

Es existieren mannigfaltige Möglichkeiten der Myombehandlung, diese reichen von einer konservativen Therapie mit Medikamentengabe bis hin zur operativen Entfernung der Gebärmutter (• Abb. 3).

\section{Therapie der Myome mit Ulipristalacetat (UPA)}

Das Präparat Ulipristalacetat mit dem Handelsnamen Esmya ${ }^{\circledR}$ gehört zu der

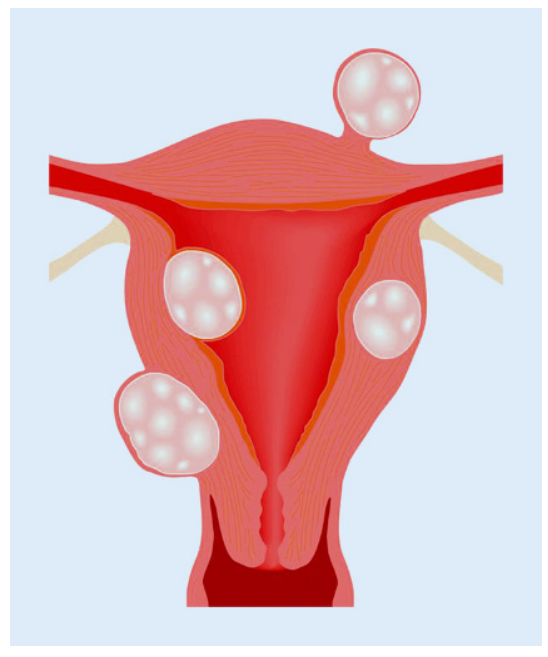

Abb. 2 ム Unterschiedliche Lokalisation der Myome

lang ist; die Ausscheidung erfolgt hauptsächlich über den Stuhl.

Nachdem in den internationalen klinischen Zulassungsstudien PEARL I und PEARL II im Jahr 2012 der Nachweis der Wirksamkeit und Sicherheit von Ulipristalacetat $5 \mathrm{mg}$ bei der Behandlung von Myomen erbracht wurde, kam es durch die EU-weite Erstzulassung von ESMYA $^{\circledR} \mathrm{zu}$ einem Paradigmenwechsel in der Myomtherapie.

Während in PEARL I die Veränderung des gesamten Myomvolumens vom Wert vor Behandlungsbeginn per MRT gemessen wurde, wurde in PEARL II die Veränderung des Volumens der drei größten Myome (Median in \%) per Ultraschall gemessen.

Dabei konnte gezeigt werden, dass es durch die Therapie mit UPA $5 \mathrm{mg}$ zu einer Verminderung des Volumens von mindestens 39\% kommt. Parallel dazu wurde das Eindämmen der Hypermenorrhoe bei über $90 \%$ aller Patientinnen im Zuge der Therapie erreicht [16, 17]. Bei jenen Patientinnen, die im Rahmen der Studie Ulipristalacetat erhielten, persistierte die Reduktion des Myomvolumens auch noch 6 Monate nach dem Absetzen der Therapie. Dazu gegensätzlich hatten bei den Patientinnen, denen das GnRH-Analogon verabreicht wurde, die Myome 6 Monate nach Therapieende nahezu wieder ihre ursprüngliche Größe erreicht. Der Effekt der Therapie mit Ulipristalacetat aufdie Lebensqualität wurde anhand eines myomspezifischen Frage- 
bogens (UFS-QOL) erhoben. Es ergab sich, dass diese bei Patientinnen mit Ulipristalacetattherapie deutlich verbessert wurde.

Dadurch, dass die Östrogenspiegel auf physiologischem Niveau blieben und daher kaum von typischen menopausalen Symptomen, wie Hitzewallungen, berichtet wurde, erzielt Ulipristalacetat einen weiteren Pluspunkt gegenüber dem GnRH-Analogon. Auch wurde Ulipristalacetat von den Patientinnen der PEARL-I und PEARL-II-Studien gut vertragen, die überwiegende Mehrzahl der Nebenwirkungen war leicht bis mittelgradig und klang spontan wieder ab. Daher war das Absetzen des Arzneimittels nicht vonnöten.

Eine Begleiterscheinung der UPATherapie wurde als „progesterone receptor modulator-associated endometrial changes" (PAEC) bezeichnet und beschreibt eine benigne Veränderung der Schleimhaut unter dem Einfluss von SPRM, wobei dies bereits mehrfach beschrieben worden ist. Das histologische Bild dieser Veränderung darf nicht mit dem einer Hyperplasie verwechselt werden. Diese bei rund $60 \%$ der Frauen vorkommenden Veränderungen sind reversibel und bilden sich spätestens 3-6 Monate nach Beendigung der Therapie zurück.

PAEC hingegen treten teilweise als Ödembildung des Stromas und teilweise als Kompaktierung in Erscheinung und kommen durch wenige Mitosen innerhalb der endometrialen Drüsen zustande.

Als Schlussfolgerung der Studien PEARL I und PEARL II ergeben sich im Hinblick auf die präoperative Vorbereitung der Patientin drei wesentliche Vorteile:

Erstens kommt es zu einer deutlichen Reduktion der durch die Myome bedingten Blutung innerhalb von 7 Tagen. Dies führt zur Stabilisierung des roten Blutbilds und damit zur besseren Vorbereitung der Patientin auf die Operation. Ferner kommt es beim Großteil der Fälle zu einer Reduzierung des Myomvolumens und dadurch zur Auswahl weniger invasiver Operationsmethoden wie Laparoskopie und Hysteroskopie. In den Fällen, wo sich die Symptome deutlich reduziert ha-

J. Gynäkol. Endokrinol. AT 2019·29:6-12 https://doi.org/10.1007/s41974-019-0083-y (c) Der/die Autor(en) 2019

\section{K. Nouri \\ Medikamentöse Therapie des Uterus myomatosus mit dem Präparat Ulipristalacetat - ein Update}

\section{Zusammenfassung}

Myoma uteri sind die am häufigsten auftretenden gutartigen Tumoren des Uterus. Die Häufigkeit des Auftretens von Myomen bei Frauen im reproduktionsfähigen Alter schwankt in der Literatur zwischen 20 und $50 \%$. Myome stellen bei einer geringen Anzahl Kinderwunschpatientinnen die alleinige Ursache für die Infertilität und Sterilität dar. Es existieren mannigfaltige Möglichkeiten der Behandlung von Myomen, sie reichen von einer konservativen Therapie bis hin zur operativen Entfernung des Uterus. Die Behandlung mit Ulipristalacetat (UPA, Esmya $^{\circledR}$ ) hat sich in den letzten Jahren gerade bei Kinderwunschpatientinnen sehr bewährt. Durch fünf Fallmeldungen von schweren Leberstörungen wurden aufgrund eines möglichen kausalen Zusammenhangs zwischen UPA und den akuten Leberversagen eine eingehende Untersuchung und eine Nutzen-Risiko-Abwägung durchgeführt. Dieses Risikobewertungsverfahren wurde nach erfolgtem EU-Kommissionsentscheid mit Juli 2018 abgeschlossen. Es beinhaltet Indikationseinschränkungen und eine neue Kontraindikation sowie Vorgaben zur notwendigen Überwachung der Leberfunktion als Maßnahme zur Risikominimierung. In dieser Arbeit wird darauf näher eingegangen und die genaue neue Anwendung der Esmya ${ }^{\circledR}$ Therapie erläutert.

Schlüsselwörter Konservative Myombehandlung · Esmya ${ }^{{ }_{-}}$ Therapie · Indikationseinschränkung · Kontraindikationen . Überwachung der Leberfunktionen

\section{Pharmacological Treatment of Uterine Fibroids with Ulipristal Aacetate: an Update}

\section{Abstract}

Myoma uteri are the most common benign tumors of the uterus. The incidence of myomas in women of reproductive age varies between 20 and $50 \%$ in the literature. Myomas are the sole cause of infertility and sterility in a small number of fertility patients. There are many options for treating myomas, ranging from conservative therapy to surgical removal of the uterus. Treatment with ulipristal acetate (UPA, Esmya ${ }^{\circledR}$ ) has proven very successful in the last few years, especially in women who wish to have children. Five case reports of severe liver disorders were performed, and a benefit-risk assessment was performed due to a possible causal relationship between UPA and acute

ben, kann Ulipristalacetat zu einer deutlichen Verbesserung der Lebensqualität führen.

In den beiden PEARL-Studien war jedoch der Einsatz von Ulipristalacetat auf 3 Monate beschränkt.

Durch die Folgestudien PEARL III, PEARL III Extension und PEARL V wurde anschließend auch die Effektivität einer Langzeittherapie mit UPA bestätigt [18]. liver failure. This risk assessment procedure was completed in July 2018 following the EU Commission decision. It includes indication restrictions and a new contraindication, as well as guidelines for the necessary monitoring of liver function as a measure for risk minimization. In this work, it will be discussed and the exact new application of Esmy $a^{\circledR}$ therapy will be explained.

\section{Keywords}

Conservative treatment of myomas Esmya ${ }^{\circledR}$ therapy · Indication restrictions Contraindications · Monitoring of liver functions

PEARL III und ihre Extension konnten zeigen, dass eine langfristige Therapie der Myome mit bis zu 4 UPA-Behandlungszyklen zu je 3 Monaten und inklusive Behandlungspausen zwischen den Therapieintervallen effektiv und arm an Nebenwirkungen ist. Die PEARL-IVStudie hat weiterhin den Einsatz von $5 \mathrm{mg}$ UPA als eine langfristige oder präoperative Therapie bestätigt - mit weniger 
Hier steht eine Anzeige.

\section{曾 Springer}




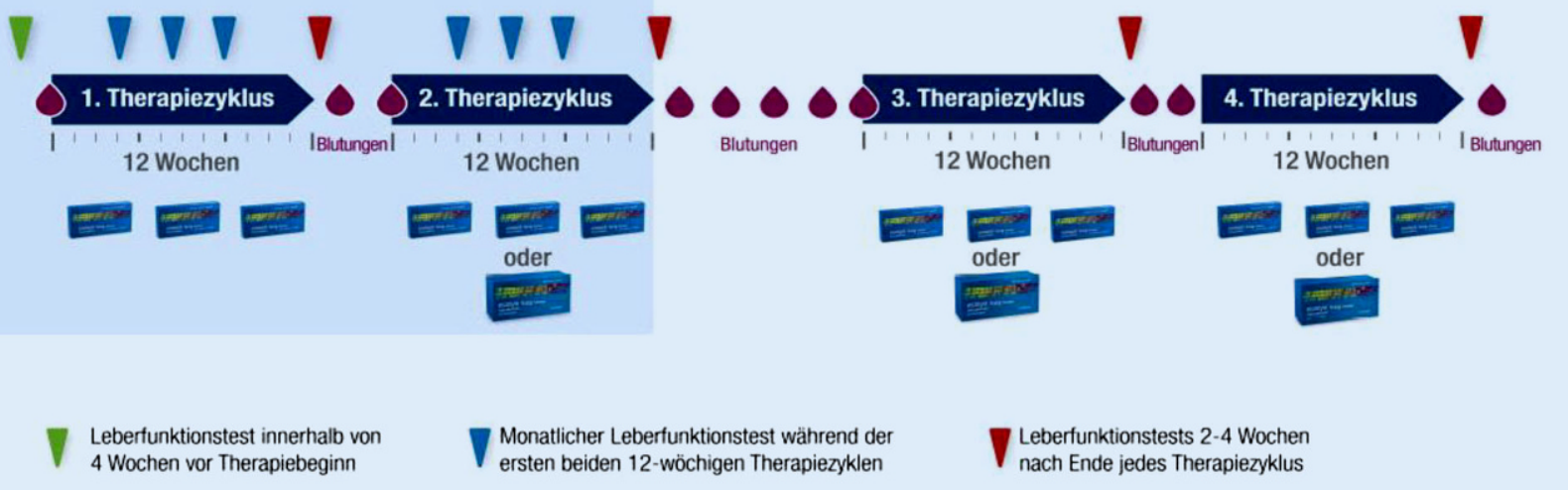

Abb. 4 \ Aktuelles Therapieschema bei Esmya ${ }^{\circledR}$. (๑ Gedeon Richter GmbH)

Nebenwirkungen als in der $10 \mathrm{mg}$-Dosisgruppe, aber gleicher Effektivität.

Während dieser Studien wurde zu unterschiedlichen Zeitpunkten eine Endometriumbiopsie durchgeführt, um die histologische Beurteilung des Endometriums im Hinblick auf PAEC durchführen zu können. Die gleiche Inzidenz von PAEC zeigt, dass eine längere Therapie mit UPA keine Erhöhung des Auftretens von PAEC mit sich bringt. Die 10-tägige Gabe von NETA konnte die Inzidenz von PAEC nicht reduzieren, führte allerdings dazu, dass die Regelblutung in der Behandlungspause früher und weniger intensiv auftrat.

Seit der Einführung von Esmya ${ }^{\circledR}$ in 2012 sind weltweit insgesamt $765.000 \mathrm{~Pa}$ tientinnen behandelt worden. Allerdings war aufgrund eines möglichen kausalen Zusammenhangs zwischen UPA und akutem Leberversagen eine eingehende Untersuchung dieses Risikos und der Auswirkungen auf das Nutzen-RisikoVerhältnis von UPA notwendig.

Fünf Fallmeldungen über schwere Leberschädigungen nach der Anwendung des Arzneimittels UPA $5 \mathrm{mg}$, von denen 4 Fälle eine Lebertransplantation erforderlich machten, waren Anlass für dieses Verfahren. Demzufolge leitete die Europäische Kommission am 30. November 2017 ein Befassungsverfahren gemäß Artikel 20 der Richtlinie 2001/726/EG aufgrund von Pharmakovigilanzdaten ein. Das europäische Risikobewertungsverfahren zum Arzneimittel Esmya ${ }^{\circledR}$ ist nach erfolgtem EU-Kommissions-
Entscheid am 26.07.2018 abgeschlossen worden [19].

\section{Zusammenfassung der Fälle}

- Fall 1 (55 J., Portugal): Die pathologischen Befunde der explantierten Leber und die früh einsetzenden unspezifischen Symptome deuten auf eine bereits vorhandene Lebererkrankung hin.

- Fall 2 (58 J., Frankreich): Die pathologischen Befunde der explantierten Leber deuten auf eine bereits vorhandene chronische hepatische Erkrankung durch eine Zirrhose hin.

- Fall 3 (45 J., Frankreich): Eine fulminante Hepatitis, verursacht durch HHV 6, kann nicht ausgeschlossen werden.

- Fall 4 (46 J., Deutschland): Eine Hepatitis-E-Infektion (HEV), die einen fulminanten Verlauf haben kann, kann nicht gänzlich ausgeschlossen werden. Die Patientin ist einige Monate nach der Lebertransplantation aufgrund einer unkontrollierbaren Sepsis durch eine Therapie mit Immunsuppressiva verstorben.

Im Verfahren wird kein definitiver Kausalzusammenhang zwischen der Anwendung von Esmya ${ }^{\circledR}$ und den schweren Leberschäden der Patientinnen hergestellt. Basierend aufdiesen Fällen ist die Annahme vertretbar, dass zumindest bis zu einer gewissen Wahrscheinlichkeit Esmya ${ }^{\circledR}$ gelegentlich zu Leberschäden beitragen kann.
Die EU-Kommissions-Entscheidung vom 26.07.2018 beinhaltet Indikationseinschränkungen, eine neue Kontraindikation sowie Vorgaben zur notwendigen Überwachung der Leberfunktion als Maßnahmen zur Risikominimierung.

\section{Durchführung von Leber- funktionstests}

- Ein Leberfunktionstest muss vor Beginn jedes Behandlungsintervalls einmal monatlich während der ersten beiden Behandlungsintervalle und 2-4 Wochen nach Beendigung der Behandlung durchgeführt werden.

- Die Behandlung mit Esmya ${ }^{\circledR}$ darf nicht begonnen werden, wenn die Transaminasen Glutamat-Oxalacetat-Transaminase (GOT/AST) oder Glutamat-Pyruvat-Transaminase (GPT/ALT) den oberen Normwert um mehr als das Zweifache überschreiten (isoliert oder in Kombination mit Bilirubin).

- Die Behandlung mit Esmya ${ }^{\circledR}$ muss abgebrochen werden, wenn die Glutamat-Oxalacetat-Transaminase (GOT/AST) oder Glutamat-Pyruvat-Transaminase (GPT/ALT) den oberen Normwert um mehr als das Dreifache überschreiten.

\section{Wie wird Esmya ${ }^{\circledR}$ angewendet?}

Die Behandlung mit Esmya ${ }^{\circledR}$ soll während der ersten Woche der Menstruationsblutung begonnen werden. Für einen Behandlungszyklus wird täglich 1 Tablet- 
te oral eingenommen. Nach 12 Wochen folgt eine Einnahmepause [20].

Wenn eine Operation aufgrund des Patientinnenwunsches und/oder aus medizinischen Gründen nicht infrage kommt, kann die Behandlung mit Esmya $^{\circledR}$ nach Absprache mit dem Frauenarzt mit einem weiteren 12-wöchigen Behandlungszyklus fortgesetzt werden. Weitere Behandlungszyklen sollten frühestens während der ersten Woche der zweiten Menstruationsblutung nach $\mathrm{Ab}$ schluss des letzten Behandlungszyklus begonnen werden (•Abb.4).

\section{Zusammenfassung}

- Esmya ${ }^{\circledR}$ bleibt weiterhin für einen Behandlungszyklus (bis zu 12 Wochen) zur präoperativen Behandlung mittlerer bis starke Symptome durch Gebärmuttermyome bei erwachsenen Frauen im fortpflanzungsfähigen Alter, für die eine Operation vorgesehen ist, indiziert.

- In Bezug auf eine Langzeitintervalltherapie mittlerer bis starker Symptome durch Gebärmuttermyome sollte eine Patientin im fortpflanzungsfähigen Alter nur dann mit mehr als einem 12-wöchigen Behandlungszyklus Esmya ${ }^{\circledR}$ behandelt werden, wenn die Patientin nicht operiert werden soll oder möchte.

\section{Neue Kontraindikation}

- Esmya ${ }^{\circledR}$ darf nicht bei Frauen mit zugrunde liegenden Lebererkrankungen angewendet werden.

\section{Korrespondenzadresse}

Assoc. Prof. PD Dr. med. univ. Kazem Nouri Universitätsklinik für Frauenheilkunde, Medizinische Universität Wien Währinger Gürtel 18-20, 1090 Wien, Österreich kazem.nouri@meduniwien.ac.at

Funding. Open access funding provided by Medical University of Vienna.

\section{Einhaltung ethischer Richtlinien}

Interessenkonflikt. K. Nouri gibt an, dass kein Interessenkonflikt besteht.

Dieser Beitrag beinhaltet keine vom Autor durchgeführten Studien an Menschen oder Tieren.

Open Access Dieser Artikel wird unter der Creative Commons Namensnennung 4.0 International Lizenz (http://creativecommons.org/licenses/by/4.0/deed. de) veröffentlicht, welche die Nutzung, Vervielfältigung, Bearbeitung, Verbreitung und Wiedergabe in jeglichem Medium und Format erlaubt, sofern Sie den/die ursprünglichen Autor(en) und die Quelle ordnungsgemäßnennen, einen Link zur Creative Commons Lizenz beifügen und angeben, ob Änderungen vorgenommen wurden.

Hinweis des Verlags. Der Verlag bleibt in Hinblick auf geografische Zuordnungen und Gebietsbezeichnungen in veröffentlichten Karten und Institutsadressen neutral.

\section{Literatur}

1. Buttram VC Jr, Reiter RC (1981) Uterine leiomyomata: etiology, symptomatology, and management. Fertil Steril 36:433-445

2. Townsend DE, Sparkes RS, Baluda MC, McClelland G (1970) Unicellular histogenesis of uterine leiomyomas as determined by electrophoresis by glucose-6-phosphate dehydrogenase. Am J Obstet Gynecol 107:1168-1173

3. Phelan JP (1995) Myomas and pregnancy. Obstet Gynecol Clin North Am 22(4):801-805

4. Lev-Toaff AS (1987) Leiomyomas in pregnancy, sonographic study. Radiology 164(2):375-380

5. Brandon et al (1998) Sex steroid receptors in human myometrium and fibroids: changes during the menstrual cycle and gonadotropin-releasing hormonetreatment. J Clin Endocrinol Metab 83:4092-4096

6. Englud et al (1993) Progesterone receptor messenger ribonucleic acid and protein are overexpressed in human uterine leiomyomas. Am JObstet Gynecol 169:78-85

7. Kawaguchi et al (1989) Mitotic activity in uterine leiomyomas during themenstrual cycle. Am J Obstet Gynecol 160:637-641

8. Harrison et al (1994) Quantification of messenger ribonucleic acid for epidermalgrowth factor in human myometrium and leiomyomatausing reverse transcriptase polymerase chain reaction. J Clin Endocrinol Metab 78:1179-1184

9. Buttram VC et al (1981) Uterine leiomyomata: etiology, symptomatology, and management. Fertil Steril 36:433-445

10. DonnezJ, JadoulP (2002)What are the implications of myomas on fertility? A need for a debate? Hum Reprod 17:1424-1430

11. Verkauf BS (1992) Myomectomy for fertility enhancement and preservation. Fertil Steril 58:1-15

12. Guo XC, Segars JH (2013) The impact and management offibroids for fertility — an evidencebased approach. Obstet Gynecol Clin North Am 39(4):521-533

13. Gambadauro $P$ (2012) Dealing with uterine fbroids in reproductive medicine. J Obstet Gynaecol 32(3):210-216
14. Kroon B, Johnson N, Chapman M et al (2011) Fibroids in infertility-consensus statement from ACCEPT (Australasian CREI Consensus Expert Panel on Trial evidence). Aust N Z J Obstet Gynaecol 51(4):289-295

15. ChwaliszK(2003) Treatment of uterineleiomyomas with the novel selective progesterone receptor modulator(SPRM). J Soc Gynecol Investig 10:301 A

16. Donnez J, Tomaszewski J, Vázquez F, Bouchard $P$, LemieszczukB, BaróF, NouriK, Selvaggi L, Sodowski K, Bestel E, Terrill P, Osterloh I, Loumaye E, PEARL II Study Group (2012) Ulipristal acetate versus leuprolide acetate for uterine fibroids. N Engl J Med 366(5):421-432

17. Donnez J, Tatarchuk TF, Bouchard P, Puscasiu L, Zakharenko NF, Ivanova T, Ugocsai G, Mara M, Jilla MP, Bestel E, Terrill P, Osterloh I, Loumaye E, PEARL I Study Group (2012) Ulipristal acetate versus placebo for fibroid treatment before surgery. N Engl J Med 366(5):409-420. https://doi.org/10. 1056/NEJMoa1103182

18. Donnez J, Vázquez F, Tomaszewski J, Nouri K, Bouchard $\mathrm{P}$, Fauser BC, Barlow DH, Palacios $S$, Donnez O, Bestel E, Osterloh I, Loumaye E, PEARL III, PEARL III Extension Study Group (2014) Long-term treatment of uterine fibroids with ulipristal acetate. Fertil Steril. https://doi.org/10.1016/j.fertnstert. 2014.02.008

19. EMA http://www.ema.europa.eu/ema/.Zugegriffen: 01.03.2019

20. Fa. Gedeon Richter. Esmya ${ }^{\circledR}$ Fachinformation (Stand:07/2018) 
Hier steht eine Anzeige.

\section{曾 Springer}

